\title{
La terminologie : un outil nécessaire pour le traducteur spécialisé
}

\section{Terminology: A necessary tool for the Specialized Translator}

\author{
Aura E. Navarro \\ University of North Georgia \\ auranavarro@gmail.com
}

\begin{abstract}
Language disciplines, including Terminology and Specialized Translation, have made great strides after the second half of the twentieth century. This development, related to technological growth and international communication that occurred during this period, has resulted in a considerable increase of concepts. Thus, experts have become more and more aware of the importance of naming these new concepts. Specialized translators were among the first language professionals to recognize the need of mastering the terminology of specialized fields in order to perform their duties well (Antia et coll., 2005). In this work, we study the very close relationship between Terminology and Specialized Translation. We also study the theoretical and practical knowledge of Terminology that a specialized translator should have.
\end{abstract}

Keywords: terminology, specialized translation, cognitive baggage, translator's skills

Depuis la deuxième moitié du $\mathrm{XX}^{\mathrm{e}}$ siècle la multiplication des communications internationales, l'intervention des États et des gouvernements en matière linguistique ainsi que l'arrivée massive des sciences et des techniques ont conduit au développement des disciplines langagières, notamment la terminologie et la traduction spécialisée. Tous ces changements ont donné lieu à une augmentation considérable des notions, raison pour laquelle les spécialistes sont devenus de plus en plus conscients de l'importance de bien nommer les nouvelles notions. D'ailleurs, à l'égard de cette préoccupation, Eugen Wüster préconisait, dès les années 1930, la normalisation des termes (Cabré, 1998). En effet, l'objectif de Wüster était de freiner la multiplication des dénominations pour une même notion dans le but de garantir une meilleure communication entre spécialistes. Wüster a ainsi constaté l'importance des terminologies univoques en communication spécialisée multilingue. Les traducteurs spécia- 
lisés, de leur côté, ont été parmi les premiers professionnels langagiers à reconnaître la nécessité de maîtriser les terminologies des domaines spécialisés afin de bien accomplir leur tâche, comme l'affirment Bassey Antia et coll., 2005.

Dans ce travail nous étudions premièrement les rapports entre la terminologie et la traduction spécialisée, rapports très étroits qui démontrent autant d'éléments communs que de différences importantes. Nous abordons par la suite les connaissances théoriques et pratiques provenant de la terminologie qui devraient faire partie intégrante du bagage cognitif et des compétences du traducteur spécialisé.

Tout d'abord, il convient de souligner le caractère polysémique du terme terminologie. En effet, celui-ci fait référence à deux définitions distinctes : a) en tant que science ou discipline, elle a " pour objet l'étude systématique, unilingue ou multilingue, de la dénomination des $<$ notions $>$ appartenant à des domaines de l'activité humaine » (Delisle, Lee-Jahnke, \& Cormier, 1999: 79) et b) en tant que vocabulaire, c'est l'ensemble des termes propres à un domaine spécialisé. Afin de les distinguer, nous utiliserons terminologie pour faire référence à la discipline et terminologies pour parler du vocabulaire d'un domaine spécialisé.

\section{RAPPORT ENTRE LA TERMINOLOGIE ET LA TRADUCTION SPECIALISÉE}

Le rapport entre la terminologie et la traduction spécialisée a été abordé par plusieurs auteurs, à savoir, Bassey Antia et coll. (2005), Ahmed Azour et Loîc Depecker (2006), María Teresa Cabré (1999), Ángela Campo (1998), Daniel Gouadec (2005), Silvia Montero et Pamela Faber (2009), Emma Rodríguez (2004) et Juan Sager (1992, 2002). Pour ces auteurs, l'importance de la terminologie dans la traduction spécialisée est amplement reconnue.

John Humbley (2011), qui reconnait également le rôle essentiel joué par la terminologie dans la traduction spécialisée, fait un état de la question de l'évolution du partenariat entre ces deux disciplines. L'auteur constate que ce partenariat, considéré comment étant évident dans les années soixante-dix du siècle dernier, a souffert des hauts et des bas depuis. Il remarque que le changement d'orientation par rapport aux buts et aux méthodes de chacune de ces disciplines est l'un des éléments causant une certaine désaffection ou rupture entre les deux disciplines, la terminologie s'étant orientée plutôt vers les recherches en intelligence artificielle. Cependant, l'auteur se réjouit de constater qu'il y a toujours des terminologues et des traducteurs qui « continuent de faire cause commune pour faire avancer les deux disciplines » et « que la recherche 
a bel et bien continué ». (Humbley, 2011). Humbley termine son article en présentant quatre nouvelles orientations favorisant la coopération entre la terminologie et la traduction, à savoir, la traduction automatique, les projets européens, la pragmaterminologie et la base ARTES (Aide à la rédaction de textes scientifiques). Ces initiatives démontrent que la période de désamour entre la terminologie et la traduction serait «bel et bien terminée », conclut Humbley ${ }^{1}$.

En outre, il existe tant d'éléments communs que de traits distinctifs entre la terminologie et la traduction. Parlons d'abord des éléments communs. D'abord, Cabré (1999) affirme que ces deux disciplines coïncident par leur nature interdisciplinaire ${ }^{2}$ étant donné qu'elles ont construit des fondements théoriques et pratiques propres à partir d'éléments provenant de la linguistique, des sciences cognitives et des sciences de la communication. La traduction spécialisée et la terminologie partagent également une longue tradition pratique, mais leurs fondements théoriques ont été établis récemment (Cabré, 1999; Sager, 2002).

D'ailleurs, tant la traduction spécialisée que la terminologie sont nées dans le but de répondre aux besoins de la communication spécialisée, soit pour fixer des concepts et des dénominations (dans le cas de la terminologie), soit pour faciliter la compréhension entre locuteurs de langues différentes (dans celui de la traduction spécialisée) (Cabré, 1999). Daniel Gouadec, pour sa part, affirme que la terminologie est née en réponse aux besoins des traducteurs et que sans traduction « il n'y aurait pas de besoins massifs en terminologies » (2005: 16). L'activité traductive a ainsi joué un rôle important dans le développement de la terminologie. Dans le monde francophone, par exemple, le courant traductionnel, comme l'appelle Pierre Auger (1985), a beaucoup influencé l'activité terminologique et a été à l'origine de la création de banques terminologiques en Europe et au Canada. Ainsi, dans des pays institutionnellement bilingues ou multilingues, des organismes internationaux ont mené des travaux dans le but d'établir des équivalences terminologiques dans les différentes langues (Cabré, 1998).

Un autre élément commun entre la terminologie et la traduction spécialisée est l'usage de deux démarches linguistiques, à savoir, les démarches sémasiologique et onomasiologique. Traditionnellement, le travail terminologique avait été orienté vers une démarche onomasiologique qui consiste à «postuler

\footnotetext{
${ }^{1}$ Pour plus de détails sur cet état de la question, voir l'article de Humbley (2011).

${ }^{2}$ Plusieurs auteurs se sont penchés sur le caractère autonome de la terminologie. Quelques théoriciens, comme Antia et coll. (2005), défendent ce caractère autonome tandis que d'autres, tel que Sager (1990), affirment plutôt le contraire. Une voie intermédiaire s'est élevée pour défendre le caractère autonome de la terminologie dans une perspective interdisciplinaire (voir Cabré, 1998 ; Rodríguez, 2004).
} 
d'abord l'existence du concept et découvrir, par la suite, les formes linguistiques servant à l'étiqueter» (L'Homme, 2005 : 1115). La démarche sémasiologique (du terme à la notion) est employée en terminologie depuis les années 1990. Cette démarche est née en rejet de l'optique onomasiologique de la terminologie « qui, pour certains, est réductrice et ne tient pas compte de toute la complexité des applications modernes de la discipline.» (L'Homme, 2005 : 1115) Quant à la traduction, les deux démarches sont employées pour expliquer le processus de traduction. Grosso modo, ce processus se divise en deux étapes : la première étape de nature sémasiologique, dans laquelle le traducteur cherche à comprendre le sens du texte source. La recherche terminologique à ce niveau vise particulièrement à cerner les notions inconnues ou imparfaitement maîtrisées (Mareschal, 1988). Ensuite, la compréhension du texte étant acquise, le traducteur entreprend la deuxième étape, de nature onomasiologique, dans laquelle il procède à la recherche et au transfert d'équivalences terminologiques et phraséologiques dans la langue cible (Mareschal, 1988).

D'autre part, il existe des différences entre la terminologie et la traduction spécialisée qui, loin d'être négatives, donnent à chaque discipline une spécificité propre. D'abord, le caractère finaliste de la traduction, face au caractère préfinaliste de la terminologie, tel que décrit par María Teresa Cabré (1998). En effet, la traduction constitue un but en soi, c'est-à-dire, un texte informatif et le produit d'un acte de discours naturel, tandis que la terminologie

n'est pas une activité pratique qui se justifie en elle-même, sinon qu'elle est destinée à satisfaire à des besoins sociaux liés soit à une communication optimale entre spécialistes et professionnels, avec ou sans l'aide de la traduction, soit au processus de normalisation d'une langue (Cabré, 1998 : 34).

En outre, plusieurs auteurs - parmi lesquels on peut mentionner Bassey Antia et coll. (2005), María Teresa Cabré (1999), Silvia Montero et Paula Faber (2009) et Juan Sager (2002) - ont souligné un rapport asymétrique entre la terminologie et la traduction spécialisée dans le sens où cette dernière nécessite des terminologies pour communiquer les connaissances spécialisées de façon adéquate et univoque tandis qu'il n'est pas nécessairement obligatoire de s'intéresser à la traduction pour faire de la terminologie.

Bref, les rapports entre la terminologie et la traduction spécialisée sont très étroits, mais dans un sens unilatéral : il est possible de faire de la terminologie sans la traduction spécialisée, mais il serait pénible de traduire un texte spécialisé sans tenir compte des différents outils terminologiques (dans la section suivante, on aborde les connaissances théoriques et pratiques de la terminologie qui sont essentielles pour le travail du traducteur spécialisé). 


\section{LA TERMINOLOGIE : UN OUTIL THÉORIQUE ET PRATIQUE POUR LA TRADUCTION SPÉCIALISÉE}

Avant d'étudier plus en détail quelles sont les connaissances tant théoriques que pratiques qui devraient faire partie intégrante du travail des traducteurs spécialisés, il convient d'évoquer la définition de compétence proposée par François Lasnier :

un savoir-agir complexe qui fait suite à l'intégration, à la mobilisation et à l'agencement d'un ensemble de capacités et d'habiletés (pouvant être d'ordre cognitif, affectif, psychomoteur et social) et de connaissances (connaissances déclaratives) utilisées efficacement, dans des situations ayant un caractère commun (Lasnier, 2000 cité par Echeverri, 2008, emphase d'Echeverri).

Dans ce sens, une compétence implique tant des connaissances déclaratives qu'opérationnelles qui s'activent dans une situation donnée. En ce qui nous concerne, nous aborderons la compétence terminologique dans la traduction spécialisée. Évidemment, la première compétence requise pour la traduction de n'importe quel type de texte est la compétence traductive $e^{3}$. Cette dernière a été abordée par des auteurs comme Jean Delisle (1992), le groupe PACTE (1998, 2002) et Jean Vienne (1998), quoique ces auteurs se penchent sur la traduction générale et ne touchent qu'assez superficiellement la traduction spécialisée. La compétence traductive fait donc partie d'un ensemble de compétences que le traducteur spécialisé doit mettre en œuvre. Cependant, elle n'est pas suffisante quand il s'agit de la traduction spécialisée. À cet égard, l'importance des connaissances terminologiques est soulignée par Montero et Faber quand elles affirment que « the adequacy of the terminology in a text as well as its suitability for the level of specialization determines to a great extent the quality of a translation » (2009: 91).

\footnotetext{
${ }^{3}$ Dans le cadre d'un projet de recherche sur la compétence en traduction, Amparo Hurtado la définit comme «le système sous-jacent de connaissances déclaratives et essentiellement opérationnelles, nécessaires pour traduire » (2008: 27). Il semble qu'il y ait un consensus parmi les différents auteurs (Cabré, 1999 ; Hurtado, 2008 ; Rodríguez, 2004, entre autres) sur les souscompétences qui composent la compétence en traduction: sous-compétence bilingue ou linguistique, sous-compétence extralinguistique, sous-compétence traductionnelle et sous-compétence instrumentale ou méthodologique. Il est aussi important de souligner que des experts du groupe EMT (European Master's in Translation) se sont également penchés sur les différentes compétences que doivent avoir les traducteurs professionnels, ainsi que les experts en communication multilingue et multimédia. Parmi les compétences mentionnées, nous retrouvons la compétence en matière de prestation du service de traduction, la compétence linguistique, la compétence technologique et la compétence interculturelle, pour ne mentionner que quelques-unes (voir EMT \& Gambier, 2009).
} 
Nous nous penchons dans ce travail sur la compétence terminologique en tant que composante essentielle du travail des traducteurs spécialisés. D'après la définition de Lasnier (2000 cité par Echeverri, 2008), la compétence terminologique est un savoir-agir complexe qui implique la mobilisation et l'agencement d'un bagage cognitif, facilitant la maîtrise de la structure conceptuelle d'un domaine de spécialité et la résolution de problèmes terminologiques dans la pratique traductive spécialisée. Cette compétence suppose donc que le traducteur spécialisé doit être étroitement familiarisé avec les connaissances de la terminologie. Comme le suggère Cabré (1999), ces connaissances sont de type théorique et pratique, raison pour laquelle nous parlerons de deux aspects : l'un théorique et l'autre pratique.

\section{ASPECT THÉORIQUE}

En tant que discipline, la terminologie se penche sur les relations entre les concepts et leurs désignations et sur la formulation de principes et de méthodes gouvernant ces relations dans un domaine spécialisé. Elle s'occupe également de la collecte et de la gestion des données terminologiques (termes, définitions, contextes, etc.). Cette gestion aboutit à des ressources terminologiques, notamment des glossaires, des bases de données, des thésaurus et des dictionnaires. Les connaissances théoriques résultant de l'activité terminologique sont nécessaires pour le travail du traducteur spécialisé pour les raisons que nous abordons ci-dessous.

D'un côté, elles facilitent la maîtrise de la structure conceptuelle d'un domaine de spécialité, structure que Seleskovitch et Lederer (2014) appellent bagage cognitif. Maîtriser cette structure conceptuelle est essentiel pour le traducteur spécialisé. En effet, sans ce bagage, le traducteur éprouvera une très grande difficulté à suivre le fil conducteur du texte source et à aboutir à une traduction de qualité. En outre, nous partageons l'avis de Cabré (1999) lorsqu'elle affirme que les termes reflètent la structure conceptuelle d'un domaine de spécialité et que chaque terme désigne un concept faisant partie de cette structure. Dans ce sens, l'usage des terminologies adéquates (univoques, claires et concises) aidera le traducteur à maintenir le degré de précision et de spécialisation que requiert la traduction spécialisée.

De l'autre côté, comme le suggère Cabré (1999), la terminologie rend compte du processus d'apprentissage et de transfert de connaissances spécialisées des experts. En effet, le travail terminologique procède d'une structure conceptuelle et, dans ce sens, seuls les spécialistes des domaines concernés peuvent bien maîtriser les connaissances spécialisées d'un domaine. Cependant, 
il convient de souligner que, bien que le traducteur ne soit pas un spécialiste, il peut progressivement en devenir $u^{4}$. Dans une approche cognitive de la traduction, Vandaele (2001) propose d'extraire les noyaux conceptuels des domaines de spécialité. Ces noyaux serviront «d'ancrages auxquels relier les notions nouvelles rencontrées dans les textes à traduire » (Vandaele, $2001: 16$ ). Un noyau conceptuel est défini comme l' « ensemble de concepts autour desquels s'organise un domaine notionnel» (Vandaele, 2001: 18). Ces noyaux sont acquis dans un processus dynamique qui rejoint le modèle naturel d'apprentissage des spécialistes, lesquels attachent les nouvelles connaissances à un bagage cognitif déjà organisé.

Il est essentiel pour le traducteur non seulement de maîtriser les termes, mais il lui faut également une appréhension globale du domaine. Grâce à une approche terminologique, le traducteur spécialisé parviendra à une vue d'ensemble d'un domaine et pourra attacher les connaissances acquises à un tout cohérent (Froeliger, 2013). Bref, les connaissances théoriques de la terminologie sont essentielles pour le traducteur car elles facilitent la maîtrise de la structure conceptuelle d'un domaine de spécialité ainsi que le processus d'apprentissage du traducteur dans le but de devenir progressivement un expert du domaine.

\section{ASPECT PRATIQUE}

En ce qui concerne l'aspect pratique, Cabré (1999) fait référence aux connaissances de la terminologie dont le traducteur spécialisé doit se servir pour la résolution de problèmes terminologiques dans la pratique traductive. Étant donné que la traduction spécialisée mène à de continuelles prises de décision, le traducteur spécialisé est amené à se forger des habitudes de travail et à développer des voies de réflexion sur les stratégies menant à la résolution de problèmes dans sa pratique traductive. La compétence terminologique renferme ainsi l'habileté de développer diverses stratégies permettant au traducteur d'aboutir plus rapidement à l'identification du type de problème terminologique auquel il fait face pendant le processus de traduction et à en trouver la solution.

Dans une perspective semblable à celle de Cabré, Michel Rochard (2008) souligne que le processus de traduction n'est pas toujours linéaire - de la compréhension à la réexpression. Au contraire, quand le traducteur affronte des

\footnotetext{
${ }^{4}$ Il existe une discussion sur le niveau de spécialisation atteint par le traducteur spécialisé. Quelques auteurs affirment que le traducteur spécialisé n'arrivera pas à avoir le même niveau de spécialisation que les experts du domaine (Azour et Depecker, 2006 ; Gallardo et Irazabal, 2002 ; Rodríguez, 2004 ; entre autres) tandis que d'autres affirment, au contraire, que le traducteur devient progressivement un expert du domaine en question (Cabré, 1999 ; Vandaele, 2001).
} 
difficultés, soit de compréhension, soit de réexpression, il fait des allers-retours entre la documentation, les outils terminologiques, la mobilisation du bagage cognitif et la mise en relation logique des éléments trouvés. Bref, le traducteur s'inscrit dans une démarche pour laquelle la mise en œuvre de sa compétence terminologique lui facilitera la résolution de différentes difficultés tout au long du processus de traduction. Dans ce contexte, la terminologie devient absolument nécessaire pour résoudre des questions pratiques de traduction.

Pour ce faire, le traducteur doit entreprendre des recherches terminologiques dans le but de satisfaire des besoins immédiats, raison pour laquelle des connaissances sur la terminologie ponctuelle lui sont nécessaires. D'après Célestin et coll. (1990), la terminologie ponctuelle ${ }^{5}$ porte sur la recherche du sens d'un terme, du terme correspondant à un concept, d'attestation d'un terme ou d'un équivalent. En tant que terminologue ponctuel, le traducteur spécialisé peut également proposer des unités néologiques, comme le suggèrent Natividad Gallardo et Amelia de Irazabal (2002), ainsi qu'Emma Rodríguez (2004). À ce niveau, le traducteur doit avoir des connaissances sur la consultation et l'évaluation des outils terminologiques, sur le processus de formation des termes et sur la collecte et l'analyse de données pour aboutir à une solution (Célestin et coll., 1990). Cependant, il faut tenir compte de ce que, par la nature immédiate de la terminologie ponctuelle, les solutions proposées par le traducteur sont plutôt provisoires. Dans un degré d'implication majeur, le traducteur peut devenir un terminologue systématique quand, au-delà de son rôle de terminologue ponctuel, il s'implique volontairement dans l'activité terminologique résultant de son activité traductive. Afin de donner un caractère plus systématique à cette activité terminologique, le traducteur devra construire sa propre base de données avec les solutions proposées au fil de ses traductions. Ceci dans le but de les faire connaître sous forme de glossaire aux autres traducteurs du même domaine (Cabré, 1990). En tant que terminologue systématique, le traducteur doit posséder des connaissances de gestion terminologique plus complètes sur le comportement des terminologies et sur la recherche et le traitement des termes, ainsi que sur les logiciels employés à ces fins.

En outre, le traducteur spécialisé doit bien connaître les ressources terminologiques dont il dispose afin d'organiser stratégiquement sa recherche (Vandaele et coll., 2008), ce que nous appellerons sous-compétence documentaire. Des auteurs tels que Michel Bédard (1986), María Teresa Cabré (1999), Heribert Picht et Jennifer Draskau (1985) s'accordent pour dire que les

${ }^{5}$ D'après Tina Célestin et coll. (1990: 17-18), la terminologie ponctuelle se penche sur un terme isolé ou sur un groupe restreint de termes, tandis que la terminologie thématique ou systématique porte sur l'ensemble des termes d'un domaine spécialisé. 
ressources terminologiques à la disposition du traducteur spécialisé appartiennent généralement à deux types. D'une part, les documents spécialisés non lexicographiques qui permettent d'acquérir des connaissances sur le domaine spécialisé et de combler des lacunes sur la structure conceptuelle du domaine en question. Dans cette première typologie, nous trouvons, entre autres, les monographies, les périodiques et les revues spécialisées, les thésaurus et les encyclopédies. Ainsi que le suggère Michel Rochard (2008), il faut également tenir compte de l'expert ou du spécialiste du domaine qui peut devenir une ressource précieuse « en apportant de nouveaux éclairages, du liant entre terme, définition, domaine, zone d'utilisation, contexte, phraséologie, traduction, etc. » (Rochard, $2008:$ 71).

D'autre part, le traducteur peut consulter les ouvrages lexicographiques généraux et spécialisés. Ces documents fournissent des informations sur les termes et leurs équivalents dans un domaine de spécialité. Dans cette deuxième typologie, nous trouvons les dictionnaires de langue générale, les dictionnaires encyclopédiques, les dictionnaires terminologiques (unilingues, bilingues et multilingues), les vocabulaires spécialisés et les banques de données terminologiques.

Aujourd'hui, les nombreux progrès technologiques permettent au traducteur spécialisé d'enrichir sa sous-compétence documentaire à l'aide des outils électroniques et informatisés. Ainsi, en faisant un simple clic, il a accès à plusieurs ressources documentaires, lexicographiques ou non, en version électronique ou en ligne qui peuvent d'ailleurs être mises à jour très rapidement. Le traducteur compte également sur Internet, une bibliothèque universelle qui a complètement changé le panorama général de l'information (Jiménez, 2002). Bref, la sous-compétence documentaire facilitera la recherche terminologique du traducteur si celui-ci est capable de bien se servir des outils terminologiques fiables et cohérents.

À ce stade, il est important de s'arrêter brièvement pour parler de l'importance de la formation professionnelle des traducteurs en matière terminologique. Quoique les cours de terminologie ne soient pas toujours inclus dans les programmes de traduction, pour Froeliger, «la science terminologique mérite une place essentielle dans l'enseignement et dans la pratique de la traduction pragmatique » (Froeliger, $2013: 93$ ).

Gouadec s'exprime lui aussi sur ce sujet en affirmant que :

« la terminologie fournit, à qui veut bien s'y arrêter, l'une des meilleures clés de compréhension des mécanismes de transfert inter-culturel et interlinguistique ou, plus précisément, des conditions de convergence/divergence entre univers et systèmes de filtres instituant les représentations de ces univers » (Gouadec, $2005: 15$ ). 
L'intégration de la terminologie dans les programmes de traduction serait donc un atout pour le traducteur spécialisé dans le sens que la connaissance terminologique lui permettra d'aboutir à une meilleure compréhension du texte source et du domaine de spécialité, puis de devenir progressivement un expert apte à trouver des solutions pour les problèmes terminologiques auxquels il fait face pendant la traduction de textes spécialisés.

\section{REMARQUES FINALES}

Le rapport entre la terminologie et la traduction spécialisée a été exploré par de nombreux auteurs. Cabré (1999) et Rodríguez (2004), par exemple, affirment que ces deux disciplines partagent une nature interdisciplinaire ainsi qu'une longue tradition pratique et des fondements théoriques établis récemment. Elles ont d'ailleurs en commun l'usage de deux démarches linguistiques, à savoir les démarches onomasiologique et sémasiologique. D'autre part, bien que le rapport entre les deux disciplines soit très étroit, il est également asymétrique dans le sens où la traduction spécialisée nécessite des terminologies pour communiquer les connaissances spécialisées, tandis que pour faire de la terminologie, il n'est pas indispensable de s'intéresser à la traduction.

$\mathrm{Au}$ fil de cet article, nous avons étudié la compétence terminologique en tant que composante nécessaire du travail des traducteurs spécialisés. Cette compétence est un savoir-agir complexe qui implique la mobilisation et l'agencement d'un bagage cognitif, facilitant ainsi la maîtrise de la structure conceptuelle d'un domaine de spécialité et la résolution de problèmes terminologiques dans la pratique traductive. Les connaissances de la terminologie qui devraient faire partie du travail des traducteurs spécialisés ont été abordées en deux aspects : un théorique et un pratique.

Le premier fait référence aux connaissances théoriques résultant de l'activité terminologique, surtout en ce qui concerne l'étude des relations entre les concepts et leurs désignations ainsi que la formulation de principes et de méthodes gouvernant ces relations dans un domaine spécialisé. Ces connaissances sont nécessaires pour le traducteur spécialisé puisqu'elles facilitent la maîtrise de la structure conceptuelle d'un domaine de spécialité ainsi que le processus d'apprentissage du traducteur dans le but de devenir progressivement un expert du domaine de spécialité. À ce propos, l'approche cognitive de la traduction (Vandaele, 2001) devient très utile puisqu'elle propose d'attacher les nouvelles connaissances à un bagage cognitif déjà organisé, tel que le modèle naturel d'apprentissage des spécialistes. 
L'aspect pratique, pour sa part, fait référence aux connaissances de la terminologie dont le traducteur spécialisé doit se servir pour la résolution de problèmes terminologiques dans la pratique traductive. Parmi ces connaissances, nous trouvons: la consultation et l'évaluation des outils terminologiques, la formation des termes, la collecte, le traitement et l'analyse de données terminologiques, les logiciels ainsi que les principes de gestion terminologique. Nous avons également souligné que la compétence terminologique renferme l'aptitude à développer diverses stratégies pour l'identification du type de problème terminologique auquel il fait face et pour trouver la solution. De plus, une sous-compétence documentaire a été abordée. Cette dernière implique que le traducteur spécialisé connaisse bien les ressources terminologiques dont il dispose. Bref, puisque la traduction est une activité cognitive complexe (Vandaele, 2007), les connaissances tant théoriques que pratiques de la terminologie sont une composante nécessaire au travail des traducteurs spécialisés. Quoique la traduction et la terminologie aient vécu des périodes d'amour et de désillusion au cours des dernières années, tel que l'affirme Humbley (2011), il est important de reconnaître qu'intégrer la terminologie aux programmes de traduction serait sans doute un atout dans la future carrière professionnelle des traducteurs apprentis.

Nous vivons dans une société de plus en plus mondialisée où la communication internationale n'est plus un choix mais une nécessité. D'ailleurs, les technologies de la communication et de l'information évoluent à une vitesse surprenante. Dans ce contexte, le traducteur spécialisé joue un rôle très important en tant que médiateur linguistique. Il est donc essentiel qu'il évolue et s'adapte afin d'être à la hauteur de ces nouveaux défis. La terminologie et les terminologies sont ainsi des outils essentiels lui permettant de communiquer la connaissance spécialisée de manière adéquate et univoque. 


\section{BIBLIOGRAPHIE}

Antia, B., Budin, G., Picht, H., Rogers, M., Schmitz, K., \& Wright, S. (2005). Shaping translation: A view from terminology research. Meta, 50(4). Récupéré de : http://www.erudit.org/revue/ meta/2005/v50/n4/019907ar.pdf le 28/07/2015. doi : 10.7202/019907ar

Auger, P. (1985). La terminologie au Québec et dans le monde, de la naissance à la maturité. Actes du sixième Colloque OLF-STQ de terminologie : l'ère nouvelle de la terminologie, Montréal, 27-59.

Azour, A., \& Depecker, L. (2006). Terminologie et traduction : quelques éléments. La Banque des mots, 72, 76-105.

Bédard, C. (1986). La traduction technique : principes et pratique. Montréal : Linguatech.

Cabré, M.T. (1998). La terminologie: théorie, méthode et applications (M. Cormier \& J. Humbley, Trad.). Ottawa : Presses de l'Université d'Ottawa.

Cabré, M.T. (1999). La terminología : Representación y Comunicación. Barcelona: Universitat Pompeu Fabra.

Campo, Á.M. (1998). La labor terminológica en la interpretación. Communication présentée lors du colloque Terminología, desarrollo e identidad nacional, La Havane.

Célestin, T., Godbout, G., \& Vachon-L'Heureux, P. (1990). Méthodologie de la recherche terminologique. Québec : Office de la langue française.

Delisle, J., Lee-Jahnke, H., \& Cormier, M.C. (1999). Terminologie de la traduction. Amsterdam : John Benjamins.

EMT (European Master's in Translation), \& Gambier, Y. (2009). Compétences pour les traducteurs professionnels, experts en communication multilingue et multimédia. Répéré de http://ec.europa.eu/dgs/translation/programmes/emt/key_documents/emt_competences_transl ators_fr.pdf le 18/01/2016.

Echeverri, Á. (2008). Énième plaidoyer pour l'innovation dans les cours pratiques de traduction. Préalables à l'innovation ? TTR, 21(1), 65-98. doi : 10.7202/029687ar

Froeliger, N. (2013). Les noces de l'analogique et du numérique : De la traduction pragmatique. Paris : Les Belles Lettres.

Gallardo, N., \& Irazabal, A. (2002). Elaboración de un vocabulario multilingüe del campo temático de la siderúrgica. In A. Alcina Caudet \& S. Gamero Pérez (Eds.), La traducción científico-técnica y la terminología en la sociedad de la información (Vol. 10, pp. 189-198). Castelló de la Plana: Universitat Jaume I.

Gouadec, D. (2005). Terminologie, traduction et rédaction spécialisées. Langages, 157, 14-24. Répéré de http://www.persee.fr/web/revues/home/prescript/article/lgge_0458-726x_2005_ num_39_157_971 le 28/07/2015.

Grupo PACTE, Hurtado, A., Beeby, A., Fernandez, O., et coll. (1998). Acquiring Translation Competence: Hypotheses and Methodological Problems of a Reseach Project. In A. Beeby, D. Ensinger, \& M. Presas (Eds.), Investigating translation (pp. 99-106). Amsterdam/Philadelphie: John Benjamins.

Grupo PACTE, Hurtado, A., Beeby, A., Fernandez, O., et coll. (2002). Grupo PACTE: une investigación empírico-experimental sobre la adquisición de la competencia traductora. In A. Alcina Caudet \& S. Gamero Pérez (Eds.), La traducción científico-técnica y la terminología en la sociedad de la informaciín (Vol. 10, pp. 125-138). Castelló de la Plana: Universitat Jaume I.

Humbley, J. (2011). Terminologie et traduction une complémentarité oubliée ? Tralogy, Session 1 - Terminology and Translation / Terminologie et Traduction. Répéré de http://lodel.irevues. inist.fr/tralogy/index.php?id=63 le 18/01/2016. 
Hurtado Albir, A. (2008). Compétence en traduction et formation par compétences. TTR, 21(1), 17-64. doi : 10.7202/029686ar

Jiménez, O. (2002). Las nuevas tareas y herramientas del traductor: aplicación en el aula de traducción técnica. In A. Alcina Caudet \& S. Gamero Pérez (Eds.), La traducción científicotécnica y la terminología en la sociedad de la información (Vol. 10, pp. 237-244). Castelló de la Plana : Universitat Jaume I.

L'Homme, M.-C. (2005). Sur la notion de "terme". Meta, 50(4), 1112-1132. doi : 10.7202/ 012064ar

Mareschal, G. (1988). Le rôle de la terminologie et de la documentation dans l'enseignement de la traduction spécialisée. Meta, 33(2), pp. 258-226. doi : 10.7202/003573ar

Montero, S., \& Faber, P. (2009). Terminological Competence in Translation. Terminology, 15(1), 88-104. doi : http://dx.doi.org/10.1075/term.15.1.05mon

Picht, H., \& Draskau, J. (1985). Terminology: An Introduction. Guilford : University of Surrey, Department of Linguistic and International Studies.

Rochard, M. (2008). Le traducteur-terminologue et l'expert, rencontre hypertextuelle. Traduire, 217, 61-77.

Rodríguez, E. (2004). Terminología y traducción: gestión de la terminología en la traducción de textos especializados. Cali: Universidad del Valle.

Sager, J.C. (1992). The Translator as Terminologist. In C. Dollerup \& A. Loddegaard (Eds.), Teaching Translation and Interpreting. Training, Talent and Experience (107-122). Amsterdam/Philadelphie: John Benjamins.

Sager, J.C. (2002). La terminología y la traducción en la sociedad de la información. In A. Alcina Caudet \& S. Gamero Pérez (Eds.), La traducción científico-técnica y la terminología en la sociedad de la información (Vol. 10, 17-43). Castelló de la Plana: Universitat Jaume I.

Seleskovitch, D. \& Lederer, M. (2014). Interpréter pour traduire. Paris : Les Belles Lettres.

Vandaele, S. (2001). Noyaux conceptuels et traduction médicale. Meta, 46(1), 6-21. doi : $10.7202 / 004533 \mathrm{ar}$

Vandaele, S. (2007). Quelques repères épistémologiques pour une approche cognitive de la traduction. Application à la traduction spécialisée en biomédecine. Meta, 52(1), 129-145. doi : 10.7202/014728ar

Vandaele, S., Raffo, M., \& Boudreau, S. (2008). Les défis de la pédagogie de la traduction spécialisée : mise en œuvre d'un site de référence en biomédecine. TTR, 21(2), 63-94. doi : 10.7202/037492ar

Vienne, J. (1998). Vous avez dit compétence traductionnelle? Meta, 43(2), 187-190. doi : 10.7202/004563ar 
